\title{
Hawking radiation and entropy in de Sitter spacetime
}

\author{
Zhao Ren ${ }^{a} \oplus$ Li Huai-Fan ${ }^{a, b}$ Zhang Li-Chun ${ }^{a}$ Wu Yue-qin ${ }^{a}$ \\ ${ }^{a}$ Institute of Theoretical Physics, Department of Physics, Shanxi Datong \\ University, Datong 037009 P.R.China \\ ${ }^{b}$ Department of Applied Physics, Xi'an Jiaotong University,Xi'an 710049 \\ P.R.China
}

\begin{abstract}
Using the analytic extension method, we study Hawking radiation of an $(n+4)$-dimensional Schwarzschild-de Sitter black hole. Under the condition that the total energy is conserved, taking the reaction of the radiation of particles to the spacetime into consideration and considering the relation between the black hole event horizon and cosmological horizon, we obtain the radiation spectrum of de Sitter spacetime. This radiation spectrum is no longer a strictly pure thermal spectrum. It is related to the change of the Bekenstein-Hawking(B-H) entropy corresponding the black hole event horizon and cosmological horizon. The result satisfies the unitary principle. At the same time, we also testify that the entropy of de Sitter spacetime is the sum of the entropy of black hole event horizon and the one of cosmological horizon.
\end{abstract}

Keywords: Hawking radiation, entropy correction, high dimensional de Sitter black hole

PACS: 04.70.Dy, 04.62.+v

\section{Introduction}

Research on the correction value of B-H entropy of black holes is one of the hot researches. There are many methods to discuss the correction value of B-H entropy [1-18]. Most people believe that the correction expression of B-H entropy of black hole is

$$
S=\frac{A}{4}+\chi \ln \frac{A}{4}+O\left(\frac{1}{A}\right)+\text { const }
$$

\footnotetext{
${ }^{\dagger}$ Corresponding author. E-mail: zhaoren2969@yahoo.com.cn
} 
where $A$ is the area of the black hole horizon, $\chi$ is a dimensionless constant. However, at present, the exact value of logarithmic term coefficient in the correction to black hole B-H entropy is not clear.

Within the appropriate range of parameters, de Sitter spacetime not only includes the balck hole horizon but also has the cosmological horizon. Both the black hole horizon and the cosmological horizon have thermal radiation. And they have different temperature. The entropy of black hole horizon and cosmological horizon satisfy the area formula [19]. For de Sitter spacetime, ones expect that the thermodynamic entropy of this spacetime is $S=A_{h} / 4+$ $A_{c} / 4[20,21]$, i.e. it is equal to the sum of the black hole horizon entropy and cosmological horizon entropy, where $A_{h}$ and $A_{c}$ are the area of black hole horizon and cosmological horizon respectively. At present, there is not good method to testify that the entropy of de Sitter spacetime is the sum of the black hole horizon entropy and cosmological horizon entropy [22].

In 2000, Parikh and Wilczek proposed the method to calculate Hawking radiation [23]. A lot of research are made using this method, their results all support the result obtained by Parikh and Wilczek[24-41].

It is well-known that a black hole can be taken as a thermodynamic system with temperature and entropy. For a spacetime that does not include a cosmological term, all state parameters of this thermodynamic system are reflected on the surface of the black hole horizon. The window where the black hole transfers information to the outside world is the black hole event horizon. However for the spacetime that contains a cosmological term, the state parameters of black hole not only embodies on the black hole horizon surface but also on the cosmological horizon surface. So the window that de Sitter spacetime transfers information to the outside world includes the black hole horizon and the cosmological horizon. Because state parameters of the black hole horizon and the cosmological horizon are de Sitter spacetime state parameters, there must have relation between the radiation of the black hole horizon and the one of the cosmological horizon[42-44]. At present, there is not such a research on the radiation spectrum of de Sitter spacetime after considering the relation of the black hole horizon and the cosmological horizon.

The organization of this work is as follows. Section 2 devotes to extending the Damour-Ruffini method[45,46] and under the condition that the total energy is conserved, taking the reaction of the radiation of particles to the spacetime into consideration and considering the relation between the black hole event horizon and the cosmological horizon, we obtain the radiation 
spectrum of de Sitter black hole. This radiation spectrum is no longer a strict pure thermal spectrum. It is related to the change in the $\mathrm{B}-\mathrm{H}$ entropy corresponding the black hole event horizon and the cosmological horizon. The result satisfies unitary principle. We also derive the coefficient of the logarithmic correction term of the B-H entropy in de Sitter black hole in section 3. We testify that the entropy of de Sitter spacetime is the sum of the entropy of black hole event horizon and one of cosmological horizon in section 4. Finally, we discuss our results in section 5. Throughout the study, the units $G=c=\hbar=1$.

\section{The radiation spectrum of $(n+4)$-dimensional Schwarzschild-de Sitter black hole}

We start with an $(n+4)$-dimensional SdS black hole solution, whose metric is $[20,21]$

$$
d s^{2}=-\left(1-\frac{r_{0}^{n+1} M}{r^{n+1}}-\lambda r^{2}\right) d t^{2}+\left(1-\frac{r_{0}^{n}+1}{r^{n+1}}-\lambda r^{2}\right)^{-1} d r^{2}+r^{2} d \Omega_{n+2}^{2},
$$

where for a positive cosmological constant $\Lambda$, we have

$$
\lambda=\frac{2 \Lambda}{(n+2)(n+3)}, \quad r_{0}^{n+1}=\frac{16 \pi}{(n+2) \Omega_{n+2}},
$$

$M$ is an integration constant, $\Omega_{n+2}$ denotes the volume of a unit $(n+2)$-sphere $d \Omega_{n+2}^{2}[20,21]$. The cosmological horizon $r_{c}$ and black hole horizon $r_{h}$ are two

positive real roots of the equation, $1-\frac{r_{0}^{n+1}}{r^{n+1}}-\lambda r^{2}=0$. The cosmological horizon is the larger one and the black hole horizon is smaller one. The cosmological horizon of the SdS solution has the Hawking temperature $T$ and the B-H entropy $S$

$$
\left.T=\frac{1}{4 \pi r_{c}}\left[(n+3) \lambda r_{c}^{2}\right)-(n+1)\right], \quad S=\frac{r_{c}^{n+2} \Omega_{n+2}}{4} .
$$

The gravitational mass of the SdS black hole $[47,48]$

$$
E=-M=\frac{r_{c}^{n+1}}{r_{0}^{n+1}}\left(\lambda r_{c}^{2}-1\right)
$$


Thermodynamic quantities correspond the cosmological horizon satisfy the first law of thermodynamics

$$
d E=T d S
$$

On the other hand, the black hole horizon $r_{h}$ in the SdS solution has also associated Hawking temperature $\tilde{T}$ and B-H entropy $\tilde{S}$

$$
\left.\tilde{T}=\frac{1}{4 \pi r_{h}}\left[(n+1)-(n+3) \lambda r_{h}^{2}\right)\right], \quad \tilde{S}=\frac{r_{h}^{n+2} \Omega_{n+2}}{4} .
$$

The Abbott and Deser (AD) mass $\tilde{E}$ of the SdS solution is [49]

$$
\tilde{E}=M=\frac{r_{h}^{n+1}}{r_{0}^{n+1}}\left(1-\lambda r_{h}^{2}\right) .
$$

Thermodynamic quantities correspond the black hole horizon satisfy the first law of thermodynamics

$$
d \tilde{E}=\tilde{T} d \tilde{S}
$$

In curved spacetime, Klein-Gordon equation of particles with rest mass $\mu_{0}$ is

$$
\frac{1}{\sqrt{-g}} \partial_{\mu}\left(\sqrt{-g} g^{\mu \nu} \partial_{\nu} \Phi\right)-\mu_{0}^{2} \Phi=0
$$

where

$$
\sqrt{-g}=\sin \theta r^{n+2} \cos ^{n} \theta \prod_{i=1}^{n-1} \sin ^{i} \theta_{i}
$$

Considering the condition that the radiation flow and outgoing flow in any fixed closed supersurface should be equal, we separate variables and derive[50]

$$
\Phi=\frac{1}{r^{\frac{n+2}{2}}} e^{i \omega t} e^{i m \varphi} R(r) S(\theta) Y_{j n}\left(\theta_{1}, \cdots, \theta_{n-1}, \phi\right),
$$

where $Y_{j n}\left(\theta_{1}, \cdots \theta_{n-1}, \phi\right)$ are the hyperspherical harmonics on the $n$-sphere that satisfy the equation

$\sum_{k=1}^{n-1} \frac{1}{\prod_{i=1}^{n-1} \sin ^{i} \theta_{i}} \partial_{\theta_{k}}\left[\left(\prod_{i=1}^{n-1} \sin ^{i} \theta_{i}\right) \frac{\partial_{\theta_{k}} Y_{j n}}{\prod_{i>k}^{n-1} \sin ^{2} \theta_{i}}\right]+\frac{\partial_{\phi} \partial_{\phi} Y_{j n}}{\prod_{i=1}^{n-1} \sin ^{2} \theta_{i}}+j(j+n-1) Y_{j n}=0$. 
The function $R(r)$ and $S(\theta)$ are then found as the solutions to the decoupled equations

$$
\begin{gathered}
\frac{1}{r^{n}} d_{r}\left(r^{n} \Delta d_{r}\left(\frac{R(r)}{r^{\frac{n+2}{2}}}\right)\right)+\left(\frac{r^{4} \omega^{2}}{\Delta}-\Lambda_{j l m}-\mu_{0}^{2}\right) \frac{R(r)}{r^{\frac{n+2}{2}}}=0, \\
\frac{1}{\sin \theta \cos ^{n} \theta} \partial_{\theta}\left(\sin \theta \cos ^{n} \theta \partial_{\theta} S\right)+\left(\Lambda_{j l m}-\frac{m^{2}}{\sin ^{2} \theta}-\frac{j(j+n-1)}{\cos ^{2} \theta}\right) S=0,
\end{gathered}
$$

where $\omega$ is the energy of radiation particles, $m$ is the projection of the radiation particle angular momentum on rotation axis, $\Delta=r^{2}\left(1-\lambda r^{2}\right)-$ $r_{0}^{n+1} M / r^{n-1}, \Lambda_{j l m}$ is the separation constant.

After the black hole radiated particles with energy $\omega, M$ in spacetime line element (2) will be replaced with $M-\omega$. Therefore, after considering the reaction of radiation to spacetime, we define the tortoise coordinate transformation $[18,51]$

$$
d r_{*}=\frac{r^{2}}{\Delta_{\omega}(r, M-\omega,)} d r
$$

Eq.(11) is reduced to as

$$
\frac{d^{2} R(r)}{d r_{*}^{2}}+\left(\omega^{2}-\frac{\Delta_{\omega}}{r^{4}} U(r)\right) R(r)=0,
$$

where

$$
U(r)=\Lambda_{j l m}+\frac{(n+2)(n+3)}{4}-\frac{(n+2)(n+4)}{4} \lambda r^{2}+\mu_{0}^{2} r^{2} .
$$

Let $r_{\omega}$ satisfies $\Delta_{\omega}\left(r_{\omega}\right)=0$, thus near $r=r_{\omega}$ Eq. (14) can be reduced to

$$
\frac{d^{2} R(r)}{d r_{*}^{2}}+\omega^{2} R(r)=0 .
$$

The solution of Eq.(15) is

$$
R(r)=e^{ \pm i \omega r_{*}}
$$

These solutions of ingoing wave and outgoing wave on surface $r=r_{\omega}$ are respectively 


$$
\begin{gathered}
\Psi_{\text {in }}=e^{-i \omega\left(t+r_{*}\right)}=e^{-i \omega v}, \\
\Psi_{\text {out }}\left(r>r_{\omega}\right)=e^{-i \omega\left(t-r_{*}\right)}=e^{-i \omega v} e^{2 i \omega r_{*}},
\end{gathered}
$$

where $v=t+r_{*}$ is Eddington-Finkelstein coordinate. From (13), near $r=r_{\omega}$ we have

$$
\ln \left(r-r_{\omega}\right)=\frac{\Delta_{\omega}^{\prime}\left(r_{\omega}\right)}{r_{\omega}^{2}} r_{*}
$$

Substituting (19) into (18), we obtain

$$
\Psi_{\text {out }}\left(r>r_{\omega m}\right)=e^{-i \omega v}\left(r-r_{\omega}\right)^{i 2 \omega r_{\omega}^{2} / \Delta_{\omega}^{\prime}\left(r_{\omega}\right)} .
$$

It is obvious that the solution of outgoing wave is singular at surface $r=r_{\omega}$. Eq.(20) only describes the outgoing particles outside surface $r_{\omega}$ and it can not describe the outgoing particles in surface $r=r_{\omega}$.

According the analytic extension method proposed in refs[18,42-46,52,53], we can obtain the outgoing wave of particle with energy $\omega$, the outgoing rate at surface $r=r_{\omega}$ is given by

$$
\Gamma_{\omega}=\left|\frac{\Psi_{\text {out }}\left(r>r_{\omega}\right)}{\Psi_{\text {out }}\left(r<r_{\omega}\right)}\right|^{2}=e^{-4 \pi \omega r_{\omega}^{2} / \Delta_{\omega}^{\prime}\left(r_{\omega}\right)}
$$

The process that the black hole radiates particles with energy $\omega$ is an integration process $[18,42-44,52,53]$, that is $\omega=\int_{0}^{\omega} d \omega^{\prime}$. So the outgoing rate that the black hole radiates particles with energy $\omega$ is

$$
\Gamma_{h}(i \rightarrow f)=\prod_{i} \Gamma_{\omega_{i}}=\exp \left[-4 \pi \int_{0}^{\omega} \frac{r_{\omega^{\prime}}^{2}}{\Delta_{\omega^{\prime}}^{\prime}\left(r_{\omega^{\prime}}\right)} d \omega^{\prime}\right] .
$$

Supposing B-H entropy difference before and after the black hole radiation is

$$
\Delta \tilde{S}=\tilde{S}\left(\tilde{E}-\omega^{\prime}\right)-\tilde{S}(\tilde{E})
$$

we have

$$
\frac{\partial(\Delta \tilde{S})}{\partial \omega^{\prime}}=\frac{\partial \tilde{S}\left(\tilde{E}-\omega^{\prime}\right)}{\partial \omega^{\prime}}, \quad d(\Delta \tilde{S})=\frac{\partial(\Delta \tilde{S})}{\partial \omega^{\prime}} d \omega^{\prime}=\frac{\partial \tilde{S}}{\partial \omega^{\prime}} d \omega^{\prime}
$$


According to the first law of thermodynamics $d \tilde{E}=\tilde{T} d \tilde{S}$, we derive

$$
d \tilde{S}=\frac{d \tilde{E}}{\tilde{T}}, \quad 1 / \tilde{T}\left(\tilde{E}-\omega^{\prime}\right)=\frac{4 \pi r_{\omega^{\prime}}^{2}}{\Delta_{\omega^{\prime}}^{\prime}\left(r_{\omega^{\prime}}\right)} .
$$

Thus

$$
\Gamma_{h}(i \rightarrow f)=\prod_{i} \Gamma_{\omega_{i}}=\exp \int d(\Delta \tilde{S})=e^{\Delta \tilde{S}}
$$

where $\tilde{S}$ is the black hole B-H entropy.

For cosmological horizon $r_{c}$, using the similar method we derive that the outgoing wave of particles with energy $\omega$ has the following outgoing rate on cosmological horizon surface

$$
\Gamma_{c}(i \rightarrow f)=\prod_{i} \Gamma_{\omega_{i}}=\exp \int d(\Delta S)=e^{\Delta S} .
$$

where $S$ is B-H entropy corresponding cosmological horizon.

\section{The correction to Bekenstein-Hawing en- tropy}

In the above calculations (26) and (27), we do not consider the factor $1 / r^{(n+2) / 2}$ in Eq.(10). After we consider this factor, from (21), (26) and (27) should be rewritten as

$$
\begin{gathered}
\Gamma_{h}(i \rightarrow f)=\frac{r_{i h}^{n+2}}{r_{f h}^{n+2}} e^{\Delta \tilde{S}}=\exp \left[\left(\frac{A_{f h}}{4}-\ln \frac{A_{f h}}{4}\right)-\left(\frac{A_{i h}}{4}-\ln \frac{A_{i h}}{4}\right)\right]=e^{\Delta \tilde{S}_{B}}, \\
\Gamma_{c}(i \rightarrow f)=\frac{r_{i c}^{n+2}}{r_{f c}^{n+2}} e^{\Delta S}=\exp \left[\left(\frac{A_{f c}}{4}-\ln \frac{A_{f c}}{4}\right)-\left(\frac{A_{i c}}{4}-\ln \frac{A_{i c}}{4}\right)\right]=e^{\Delta S_{B}} .
\end{gathered}
$$

Thus we derive formulas for the first order correction to B-H entropy corresponding black hole horizon and cosmological horizon.

$$
\tilde{S}_{B}=\frac{A_{h}}{4}-\ln \frac{A_{h}}{4},
$$


and

$$
S_{B}=\frac{A_{c}}{4}-\ln \frac{A_{c}}{4}
$$

where $A_{h}$ and $A_{c}$ are the black hole horizon area and the cosmological horizon area respectively. From (31), logarithmic correction term coefficient of B-H entropy in de Sitter black hole is -1 .

\section{The entropy of de Sitter spacetime}

Taking the black hole horizon and the cosmological horizon as a independent thermodynamic system, the outgoing rate that this system radiates particles with energy $\omega$ is obtained by Eqs.(28) and (29) respectively. So, the outgoing rate that de Ditter spacetime radiates particles with energy $\omega$ should be

$$
\Gamma=\Gamma_{h} \Gamma_{c}=e^{\Delta \tilde{S}_{B}+\Delta S_{B}} .
$$

In quantum mechanics we obtain the probability that the system transfers from initial state to final state is

$$
\Gamma(i \rightarrow f)=\left|M_{f i}\right|^{2} \cdot(\text { phase space factor })
$$

The first term in the right side is the square of the amplitude, and the phase space factor can be written as

$$
\text { phase space factor }=\frac{N_{f}}{N_{i}}=\frac{e^{S_{f}}}{e^{S_{i}}}=e^{\Delta S},
$$

where $N_{f}$ and $N_{i}$ are the number of microstates of final state and initial state of the system respectively. For a black hole, the number of microstate is derived from final and initial Bekenstein-Hawking entropies. So Eq.(34) is the probability that de Sitter spacetime transfers from initial state to final state after radiating particles with energy $\omega$. Therefore we obtain that the entropy of de Sitter spacetime is the sum of the entropy of black hole event horizon and one of cosmological horizon.

\section{Conclusion}

In de Sitter spacetime both the black hole horizon and the cosmological horizon have particle radiations. The researches on quantum tunneling of these 
kinds spacetimes, Refs. [54-57] considered that the black hole horizon and the cosmological horizon are independent and discussed the radiation spectrums respectively. They did not consider the relation of these two horizons.

Since de Sitter spacetime has the black hole horizon and the cosmological horizon, the state parameters describing two horizons are the same, the radiation of two horizons are related. To discuss the radiation spectrum of de Sitter spacetime, we must consider the relation of these two horizons. In this paper, we obtain the Hawking radiation spectrum considering the relation of these two horizons and obtain the result that the entropy of de Sitter spacetime is the sum of the entropy of black hole event horizon and one of cosmological horizon. This is a meaningful work for understanding the thermodynamic properties of de Sitter spacetime with black hole event horizon and cosmological horizon.

\section{ACKNOWLEDGMENT}

This work was supported by the Natural Science Foundation of Shanxi Province, China (Grant No. 2006011012) and the Shanxi Datong University doctoral Sustentation Fund,China.

\section{References}

[1] A. J. M. Medved and E. C. Vagenas, Phys. Rev. D 70, 124021(2004)

[2] A. Chatterjee and P. Majumdar, Phys. Rev. Lett. 92, 141301(2004)

[3] R. K. Kaul and P. Majumdar, Phys. Rev. Lett. 84, 5255(2000)

[4] G. A. Camellia, M. Arzano and A. Procaccini, Phys. Rev. D 70, 107501(2004)

[5] A. Chatterjee and P. Majumdar, Phys. Rev. D 71, 024003(2005)

[6] Y. S. Myung, Phys. Lett. B 579, 205(2004)

[7] R. Zhao and S. L. Zhang, Phys. Lett. B 641, 318 (2006)

[8] R. Zhao and S. L. Zhang, Phys. Lett. B 641, 208 (2006)

[9] A. J. M. Medved, E. C. Vagenas, Mod. Phys. Lett. A 20, 1723 (2005) 
[10] M. R. Setare, Eur. Phys. J. C 49, 865(2007)

[11] M. R. Setare, Phys. Rev. D 70, 087501(2004)

[12] R. Banerjee and B. R. Majhi, J. High Energy Phys. 0806, 095(2008)

[13] R. Banerjee and B. R. Majhi, Phys. Lett. B 662, 62(2008)

[14] J. Y. Zhang , Phys. Lett. B 668, 353 (2008)

[15] A. Ghosh and P. Mitra, Phys. Rev. D 71, 027502(2005)

[16] S. Hod, Class. Quant. Grav. 21, L97(2004); arXiv:hep-th/0405235

[17] A. J. M. Medved, Class. Quant. Grav. 22, 133(2005)

[18] L. C. Zhang, Y. Q. Wu, H. F. Li and R. Zhao, Europhysics Letters. 86, $59002(2009)$

[19] R. G. Cai, Nucl. Phys. B 628, 375(2002)

[20] R. G. Cai, J. Y. Ji and K. S. Soh, Class. Quant. Grav. 15, 2783(1998)

[21] M. Urano and A. Tomimatsu, Class. Quant. Grav. 26, 105010(2009)

[22] R. G. Cai, Physics 34, 555(2005)

[23] M. K. Parikh and F. Wilczek, Phys. Rev. Lett. 85, 5042(2000)

[24] E. C. Vagenas, Phys. Lett. B 503, 399(2001)

[25] E. C. Vagenas, Phys. Lett. B 533, 302(2002)

[26] E. C. Vagenas, Phys. Lett. B 559, 65(2003)

[27] M. R. Setare and E. C. Vagenas, Phys. Lett. B 584, 127(2004)

[28] M. R. Setare and E. C. Vagenas, Int. J. Mod. Phys. A 20, 7219(2005)

[29] R. Kerner and R. B. Mann, Phys. Rev. D 75, 084022(2007)

[30] R. Kerner and R. B. Mann, Phys. Lett. B 665, 277(2008)

[31] R. Zhao, H. F. Li and S. Q. Hu, Chinese Journal of Physics 45, $32(2007)$ 
[32] M. Arzano, A. J. M. Medved and E. C. Vagenas, J. high Energy Phys. 0509, 037(2005)

[33] J. Y. Zhang and Z. Zhao, Phys. Lett. B 618, 14(2005)

[34] Q. Q. Jiang, S. Q. Wu and X. Cai, Phys. Rev. D 75, 064029(2007)

[35] J, Y, Zhang and J. H. Fan, Phys. Lett. B 648, 133(2007)

[36] R. Li and J. R. Ren, Phys. Lett. B 661, 370(2008)

[37] J. J. Peng and S. Q. Wu, Phys. Lett. B 661, 300(2008)

[38] J. Y. Zhang and Z. Zhao, J. High Energ. Phys. 10, 055(2005)

[39] J. Y. Zhang and Z. Zhao, Nucl. Phys. B 725, 173(2005)

[40] K. Lin and S. Z. Yang, Phys. Lett. B 674, 127 (2009)

[41] K. Lin and S.Z. Yang, Phys. Rev. D 79, 064035 (2009)

[42] H. F. Li, S. L. Zhang, Y. Q. Wu, L. C. Zhang and R. Zhao. Eur. Phys. J. C 63, 133 (2009)

[43] R. Zhao, L. C. Zhang, H. F. Li and Y. Q. Wu, Eur. Phys. J. C 65, 289(2010)

[44] R. Zhao, L. C. Zhang, H. F. Li, Gen. Relativ. Gravit. 42, 975(2010)

[45] T. Damour and R. Ruffini, Phys. Rev. D 14, 332(1976)

[46] S. Sannan, Gen. Relativ. Gravit. 20, 239(1988)

[47] V. Balasubramanian, J. de Boer and D. Minic, Phys. Rev. D 65, 123508(2002)

[48] A. M. Ghezelbash and R. B. Mann, J. high Energ. Phys. 0201, 005(2002)

[49] L. F. Abbott and S. Deser, Nucl. Phys. B 195, 76(1982)

[50] H. Kodama, R. A. Konoplya and A. Zhidenko, Phys. Rev. D 79, 044003(2009) 
[51] J. Doukas, H. T. Cho, A. S. cornell and W. Naylor, Phys. Rev. D 80, 045021(2009)

[52] X. K. He and W. B Liu, Phys. Lett. B 653, 330 (2007)

[53] S. W. Zhou and W. B. Liu, Phys. Rev. D 77, 104021 (2008)

[54] D. Y. Chen, Q. Q. Jiang and X. T. Zu, Phys. Lett. B 665, 106(2008)

[55] S. Hemming and E. K. Vakkuri, Phys. Rev. D 64, 044006(2001)

[56] M. K. Parkh, Phys. Lett. B 546, 189(2002)

[57] A. J. M. Medved, Phys. Rev. D 66, 124009(2002) 